East African Medical Journal Vol. 77 No. 4 April 2000

HIV AND TUBERCULOSIS IN A RURAL HOSPITAL IN KENYA

J. K. Torrens MRCP, DTM\&H, Physician, PCEA Chogoria Hospital, P.O. Box 35, Chogoria, Kenya

Request for reprints to: Dr. J. K. Torrens, Department of Infectious Diseases, Seacroft Hospital, York Road, Leeds LS14 6UH, England, UK.

\title{
HIV AND TUBERCULOSIS IN A RURAL HOSPITAL IN KENYA
}

\section{J. K. TORRENS}

\begin{abstract}
Objective: To document the incidence and prevalence of HIV infection and TB in patients attending a rural Kenyan hospital.

Design: A retrospective analysis of newly diagnosed HIV and TB-infected patients, HIV and TB-related admissions, and results of blood donor screening for HIV from 1993 to 1997.

Setting: PCEA Chogoria Hospital, Eastern Province, Kenya.

Subjects: Patients diagnosed with HIV infection and/or TB; all blood donors.

Intervention: Diagnosis of pulmonary TB by Ziehl-Neelsen staining of sputum smears; diagnosis of smear negative and extrapulmonary TB based on consistent clinical and radiological features; HIV- 1 and HIV-2 testing of patients clinically suspected to be infected and all blood donors.

Main outcome measures: Patients diagnosed with HIV and/or TB from 1993 to 1997; the number of HIV and TB related admissions and the associated mortality rates.

Results: A rising incidence of newly diagnosed HIV patients is documented, and an increasing number of TB patients are co-infected with HIV. The number of HIV inpatient episodes is increasing, against a background of falling inpatient and outpatient episodes. HIV seroprevalence among blood donors is stable at $3-4 \%$. The proportion of TB patients suffering from extrapulmonary $\mathrm{TB}$ is increasing $(\mathrm{p}=\mathbf{0 . 0 1 1})$, probably as a result of the increase in HIV.

Conclusion: The HIV epidemic is having an increasing impact on rural Kenyans' health, although background seroprevalence rates are apparently stable. TB patients co-infected with HIV are placing a growing burden on health care resources. All health care facilities face a challenge as to how best to use limited resources to combat both these deadly diseases.
\end{abstract}

\section{INTRODUCTION}

The enormous burden of human immunodeficiency virus (HIV) infection in sub-Saharan Africa has been well documented(1). Seventy per cent (22.5 million) of the world's infected patients live in this region, the majority of whom have no hope of benefitting from the highly active (and highly expensive) antiretroviral therapy (HAART), which has dramatically reduced hospital admission rates in developed countries(2). In Africa, the HIV epidemic not only causes substantial morbidity and mortality for infected individuals, but also creates major social and economic problems for the wider community. One concern is the impact the epidemic is having on local health care facilities, including hospital bed occupancy rates(3). Another related area of concern is the effect of HIV infection on the incidence of tuberculosis (TB). Studies have shown a clear link between HIV and increased rates of TB infection, with some also indicating higher relapse rates in dually infected individuals(4). Many of these studies have been based on urban populations where the prevalence of HIV tends to be higher, but it is important to know what impact the epidemic is having on rural communities also. The Presbyterian Church of East Africa (PCEA) Chogoria Hospital is situated on the eastern side of Mount Kenya, in Eastern Province. The hospital's catchment area is approximately $3000 \mathrm{~km}^{2}$, and is mainly rural in nature. Most of the population live and work locally, but a substantial minority (predominantly male) work in Nairobi, returning home at the weekends or less frequently. The data presented here are reviewed to assess the impact of the HIV epidemic on the local population, and the hospital itself, over a five year period. Data concerning new cases of TB and TB- related admissions are also examined, to see if there have been changes over the same period.

\section{MATERIALS AND METHODS}

At PCEA Chogoria Hospital, TB is diagnosed and treated in accordance with Kenya's National TB and Leprosy Programme, through which antituberculosis drugs are provided free of charge. The hospital's laboratory is equipped to perform microscopy and Ziehl-Neelsen staining of sputum and other specimens. HIV- 1 and HIV-2 antibody testing is carried out by the Capillus latex aggregation test kit (Cambridge Diagnostics Ireland, Galway, Ireland), following pre-test counselling and the verbal consent of 
patients suspected to be infected. Post-test counselling is offered to those individuals whose results are positive. In addition, since 1995 all TB patients have been counselled and tested for HIV infection, once verbal consent has been obtained. All blood donors were routinely tested for HIV during the five years reviewed here; if consent was withheld, no blood was donated by that person.

The hospital's computerised database records the number of inpatient episodes, each coded by diagnosis. It also records the outcome for each episode. Separate written registers are kept for outpatient episodes and newly diagnosed HIV and TB patients, providing a specific record of these cases. A retrospective review of all patients either admitted or diagnosed with HIV or TB was carried out, from 1993 to 1997, by examining the computerised database and the written registers. The results of all blood donors tested for HIV over the same period were also reviewed. In view of the relatively crude nature of the data and a number of potentially confounding variables, statistical analysis was performed on only a few of the parameters recorded. Where this was possible, a simple Chi square test was used.

\section{RESULTS}

The number of patients presenting with a new diagnosis of HIV infection and/or TB, from 1993 to 1997 (Table 1) has increased from 95 to 315 . This is despite a decrease in the total number of outpatient and medical inpatient episodes since 1994. An increasing proportion (from seven per cent in 1995 to $22 \%$ in 1997) of newly diagnosed TB cases (Table 1) are co-infected with HIV. The total number of new TB patients fluctuated over the five-year period, but there is a statistically significant increase in the proportion due to extrapulmonary disease. This rises from $6.4 \%$ to $16.7 \%$ between 1994 and $1997(\mathrm{p}=0.011)$. There was no significant change in the proportion of pulmonary TB patients with smear negative disease, although specific data are only available from 1995 to 1997 . The seroprevalence of HIV infection among blood donors ranged from five to three per cent over the five years, but overall is fairly constant.

Table 1

Details of patients newly diagnosed with HIV and/or TB from 1993 to 1997

\begin{tabular}{|c|c|c|c|c|c|}
\hline & 1993 & 1994 & 1995 & 1996 & 1997 \\
\hline HIV & 95 & 126 & 222 & 271 & 315 \\
\hline \multirow[t]{2}{*}{$\mathrm{PTB}^{1}$ smear +ve } & & 160 & & & \\
\hline & Not known & (all PTB) & 108 & 129 & 111 \\
\hline \multicolumn{6}{|l|}{ PTB smear -ve } \\
\hline [\% all PTB] & Not known & & $63(36.8 \%)$ & $101(43.9 \%)$ & $68(38 \%)$ \\
\hline \multicolumn{6}{|l|}{ Extrapulm TB } \\
\hline [\% of all TB] & Not known & $11(6.4 \%)$ & $21(10.9 \%)$ & $41(15.1 \%)$ & $36(16.7 \%)$ \\
\hline TB (Total) & 145 & 171 & 192 & 271 & 215 \\
\hline \multicolumn{6}{|l|}{$\%$ TB cases } \\
\hline with HIV & Not known & Not known & $7 \%$ & $17 \%$ & $22 \%$ \\
\hline \multicolumn{6}{|l|}{$\%$ blood donors } \\
\hline with HIV & $4.6 \%$ & $3 \%$ & $5 \%$ & $3.5 \%$ & $3.2 \%$ \\
\hline \multicolumn{6}{|l|}{ Outpatient } \\
\hline episodes & 62913 & 70828 & 66944 & 62199 & 54160 \\
\hline
\end{tabular}

1 pulmonary tuberculosis

Table 2

Details of medical inpatient episodes diagnosed as HIV or TB from 1993 - 1997

\begin{tabular}{|c|c|c|c|c|c|}
\hline & 1993 & 1994 & 1995 & 1996 & 1997 \\
\hline HIV & 39 & 49 & 91 & 107 & 138 \\
\hline$(\%)^{1}$ & $(1.2 \%)$ & $(1.2 \%)$ & $(2.2 \%)$ & $(3 \%)$ & $(4 \%)$ \\
\hline HIV mortality (\%) & $28.2 \%$ & $18.4 \%$ & $39.6 \%$ & $39.3 \%$ & $42.8 \%$ \\
\hline TB & 90 & 69 & 41 & 78 & 113 \\
\hline$(\%) 1$ & $(2.7 \%)$ & $(1.7 \%)$ & $(1 \%)$ & $(2.2 \%)$ & $(3.3 \%)$ \\
\hline TB mortality (\%) & $17.8 \%$ & $1.5 \%{ }^{2}$ & $39 \%$ & $28.2 \%$ & $17.7 \%$ \\
\hline Inpatient episodes & 3357 & 4168 & 4057 & 3581 & 3425 \\
\hline
\end{tabular}

${ }^{1}$ Percentage of all medical inpatient episodes

${ }^{2}$ See results section 
The number of HIV inpatient episodes (Table 2) shows a yearly increase, against an overall background of decreasing medical unit admissions since 1994. The HIV mortality rate has been stable at approximately $40 \%$ from 1995 to 1997; analysing the figures from 1993 to 1997 fails to detect any statistically significant increase in mortality ( $\mathrm{p}=0.076$ ). The number of TB inpatient episodes, and the associated mortality rate, has remained relatively stable over the study period. The mortality rate for TB inpatients in 1994 is very low in comparison with other years. Every effort has been made to ensure the data reported here are complete, but it may be that some mortality details are missing from 1994 to account for this apparent anomaly.

\section{DISCUSSION}

These results of this study show that, from 1993 to 1997, a growing number of individuals have been diagnosed with HIV infection at PCEA Chogoria Hospital. This is perhaps not surprising, but it assumes more significance when set against the background of declining outpatient and inpatient numbers attending the hospital. Also, the proportion of blood donors testing positive for HIV has remained relatively constant over the five year period. This raises the possibility that the HIV epidemic may be stabilising, at least with respect to the incidence of newly acquired infection. Further evidence of this has been recently reported from a survey of antenatal patients attending clinics in Nairobi(5). If this is indeed the case, this data would suggest that the increasing number of patients being diagnosed at Chogoria is because of the natural progression and presentation of the disease in individuals infected some years previously. On the other hand, the seroprevalence figures in blood donors may be an underestimate of the true prevalence. It is widely known in the community that blood donated at Chogoria is tested for HIV, and this may be a deterrent to some who either know or suspect their HIV status to be positive. However, an anonymous survey of 243 antenatal patients carried out at Chogoria in 1996 (unreported) found a similar seroprevalence of $4.1 \%$, but this may also be a falsely low figure. HIV infected women suffer from decreased fertility and increased rates of foetal loss, thus affecting the number attending antenatal clinics $(6,7)$.

The number of HIV related medical inpatient episodes has increased year on year, although as only those patients suspected of HIV infection were tested, the results obtained are likely to be an underestimate of the true figure. Gilks et al(3) reported that, in 1992, 39\% of acute adult medical admissions to Kenyatta National Hospital, Nairobi were HIV positive. They also reported a mortality of $35 \%$ in these HIV infected patients, which is similar to the mortality of approximately 40\% seen at Chogoria from 1995 to 1997.

The figures with respect to TB are difficult to analyse accurately. Patient consultation and admission rates are likely to have been affected by a number of potentially confounding factors, such as the introduction of Directly
Observed Therapy, Short-course (DOTS) in 1993, rising hospital fees and an increased choice of health care facilities in the local area. These factors might be expected to have a greater influence on TB patients who are HIV negative, particularly if they are relatively well, than sicker HIV positive patients, although this cannot be substantiated. However, there does appear to be an increase in the proportion of newly diagnosed TB patients co-infected with HIV, although the number of TB cases who either refused testing or were not tested is unknown. In 1997, $22 \%$ of newly diagnosed TB patients were HIV infected; this is similar to the prevalence of $26.5 \%$ recorded at the Infectious Disease Hospital, Nairobi, 1997(8), but lower than the 60\% in Zambia in 1990(9). There has been a statistically significant increase $(p=0.011)$ in the number of patients with extrapulmonary $\mathrm{TB}$, as a proportion of all TB cases. This may well reflect the rising prevalence of HIV co-infection in the TB cases, as extrapulmonary disease is more common in these patients(4). Unfortunately, it is not possible to say, from these data, exactly how many of the HIV positive patients infected with TB presented with extrapulmonary disease. Overall, since 1993 the percentage of medical inpatient episodes due to TB has not changed dramatically, and neither has the mortality rate, if one ignores the suspect figure for 1994. It might have been expected, given the increasing proportion of TB patients found to be co-infected with HIV, that the mortality rate of these cases would be higher and thus affect the overall figure for TB inpatients. However, our data support the finding of Brindle et al (10), that the increased mortality in HIV positive patients co-infected with TB may be due to pathology other than TB itself. It is not possible to report, from the Chogoria data, how many of the deaths in TB patients occurred in those co-infected with HIV.

In conclusion, the consequences of HIV infection are having a growing impact on this part of rural Kenya. This is despite an apparently stable background seroprevalence. Malaria remains the commonest medical inpatient diagnosis at Chogoria, but the rising number of HIV infected patients requiring admission is likely to have an increasing and disproportionately higher impact on the amount of resources used. There also appears to be an increasing proportion of TB cases co-infected with HIV. Both these facts have serious implications not only for the health of the local population, but also in terms of health care resources. A united effort between the local community and those providing health care to the community is vital, if progress is to be made in addressing these life-threatening challenges.

\section{ACKNOWLEDGEMENTS}

To Kiara Bundi and Charity Mugambi for assistance with data collection, and the Medical Officer in charge of the hospital for permission to publish these data..

\section{REFERENCES}

1. Msamanga G.I. and Fawzi W.W. The double burden of HIV infection and tuberculosis in subSaharan Africa. N. Engl. J. Med. 1997; 337:849-851. 
2. Palella F.J. Jr, Delaney K.M., Moorman A.C., Loveless M.O., Fuhrer J. and Satten G.A. et al.Declining morbidity and mortality among patients with advanced human immunodefeciency virus infection. N. Engl. J. Med. 1998; 338:853-860.

3. Gilks C.F., Floyd K., Otieno L.S., Adam A.M., Bhatt S.M. and Warrell D.A. Some effects of the rising case load of adult HIVrelated disease on a hospital in Nairobi. AIDS. Hum. Retrov. 1998; 18:234-240

4. Harries A.D. Tuberculosis in HIV-infected persons with special emphasis on sub-Saharan Africa. J. Infect. 1998; 37:205-209.

5. Jackson D.J., Ngugi E.N., Plummer F.A., Kirui P., Kariuki C. and Ndinya-Achola J.O.et al.Stable antenatal HIV-1 seroprevalence with high population mobility and marked seroprevalence variation among sentinel sites within Nairobi, Kenya. AIDS. 1999; 13:583-589.

6. Gray R.H., Wawer M.J., Serwadda D., Sewankambo N., Li C. and Wabwire-Mangen F. et al.Population-based study of fertility in women with HIV- 1 infection in Uganda.Lancet. 1998;351:98103.

7. Boisson E., Nicoll A., Zaba B. and Rodrigues L.C. Interpreting HIV seroprevalence data from pregnant women. AIDS. Hum. Retrov. 1996; 13:434-439

8. Nunn P., Gicheha C., Hayes R., Gathua S., Brindle R. and Kibuga D. et al. Cross-sectional survey of HIV infection among patients with tuberculosis in Nairobi, Kenya. Tubercle Lung Dis. 1992; 73:45-51.

9. Elliot A.M., Nkandu L., Tembo G., Halwiindi B., Steenbergen G. and Machiels L. et al. Impact of HIV on tuberculosis in Zambia: a cross sectional study. Brit. Med. J. 1990; 301:412-415.

10. Brindle R.J., Nunn P.P., Batchelor B.I.F., Gathua S.N., Kimari J.N. and Newnham R.S. et al. Infection and morbidity in patients with tuberculosis in Nairobi, Kenya. AIDS. 1993; 7:1469-1474. 